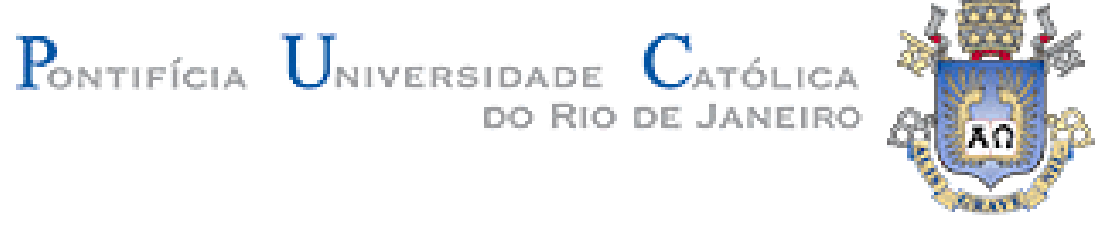

Daniel Oswaldo Santana de Souza

O Medo de Errar no Ambiente de Trabalho

Dissertação apresentada ao Programa de Pósgraduação em Administração de Empresas da PUCRio como requisito parcial para a obtenção do titulo de Mestre em Administração de Empresas.

Orientadora: Prof ${ }^{a}$. Patricia Amélia Tomei

Rio de Janeiro

Abril de 2012 
Daniel Oswaldo Santana de Souza

\title{
O Medo de Errar no Ambiente de Trabalho
}

\begin{abstract}
Dissertação apresentada como requisito parcial para obtenção do grau de Mestre pelo Programa de Pósgraduação em Administração de Empresas da PUC-Rio. Aprovada pela Comissão Examinadora abaixo assinada.
\end{abstract}

Profa. Patrícia Amélia Tomei

Orientadora

Departamento de Administração - PUC-Rio

Profa. Alessandra de Sá Mello da Costa

Departamento de Administração - PUC-Rio

Prof. Giuseppe Maria Russo

Profa. Mônica Herz

Vice-Decana de Pós-Graduação do CCS

Rio de Janeiro, 12 de abril de 2012 
Todos os direitos reservados. É proibida a reprodução total ou parcial do trabalho sem a autorização da universidade, do autor e da orientadora.

Daniel Oswaldo Santana de Souza

Jornalista, 25 anos, formado pela PUC-Rio.

Ficha Catalográfica

Souza, Daniel Oswaldo Santana de

O medo de errar no ambiente de trabalho / Daniel Oswaldo Santana de Souza ; orientadora: Patricia Amélia Tomei. - 2012.

70 f. ; $30 \mathrm{~cm}$

Dissertação (mestrado)-Pontifícia Universidade Católica do Rio de Janeiro, Departamento de Administração, 2012.

Inclui bibliografia

1. Administração - Teses. 2. Medo de errar. 3. Erro. 4. Medo organizacional. I. Tomei, Patricia Amélia. II. Pontifícia Universidade Católica do Rio de Janeiro. Departamento de Administração. III. Título.

CDD: 658 
À meus pais... 


\section{Agradecimentos}

Agradeço a Deus por tudo.

Principalmente pelos meus pais, Bernadete e Oswaldo, demonstração máximo de Seu infinito amor por mim. Por minha tia Mary e minha irmã Damaris, por juntas completarem o meu núcleo familiar, sustentador principal de tudo que sou, de tudo que eu ambiciono e de tudo que eu venho a realizar.

E por todos os meus amigos, sem os quais minha rotina certamente não teria graça alguma.

Agradeço a todos os integrantes da banca, professora Patrícia Tomei, professora Alessandra Costa e professor Giuseppe Russo, pela orientação e por todo o auxílio na concepção deste trabalho.

Agradeço especialmente ao professor Jorge Ferreira, pela confiança e por todo o conhecimento compartilhado. Atribuo a ele boa parte das grandes conquistas do curso, resultantes de seus constantes desafios intelectuais. Muito, muito obrigado. 


\section{Resumo}

Souza, Daniel Oswaldo Santana de; Tomei, Patricia Amélia. O Medo de Errar no Ambiente de Trabalho. Rio de Janeiro, 2012. 70p. Dissertação de Mestrado - Departamento de Administração, Pontifícia Universidade Católica do Rio de Janeiro.

Muitos são os fatores que contribuem para a disseminação do sentimento do medo no ambiente de trabalho. Mudanças tecnológicas e constantes transformações nos sistemas de gestão têm aumentado as pressões por excelência, aliadas à produtividade, intensificando dessa forma a experiência profissional (CUNHA, 2006). Tal fato, na maioria dos casos, é percebido pelos pesquisadores organizacionais como algo danoso, capaz de comprometer a integridade psicológica e até física de quem o vivencia, influenciando negativamente o funcionamento organizacional (SUAREZ, 1993; APPLEBAUM, 1998). Todavia, há quem veja no medo algo positivo. Tal sentimento, por vezes, se bem gerido, é defendido como um ponto sustentador da interação social (KOURY, 2002) ou até como um potencializador de performance, contribuindo para o bom desempenho de gestores e funcionários numa empresa. O objetivo principal desta dissertação é entender como variáveis demográficas - tais como idade, gênero, status profissional, tipo de vínculo profissional e nível hierárquico - influenciam na intensidade do medo de errar no ambiente organizacional. Para tanto, foi aplicado o questionário Performance Failure Appraisal Inventory - PFAI (CONROY, 2002) a uma amostra intencional, não probabilística, escolhida por acessibilidade de alunos de cursos de pós-graduação em Administração de Empresas de uma universidade carioca. Com base no tratamento estatístico dos dados observou-se o fenômeno do "falso baixo", que coloca em evidência as limitações relacionadas à expressão de sentimentos, principalmente de sentimentos negativos, tal como o medo. Apesar dos baixos índices de medo de errar aferidos, os dados estatísticos descritivos rechaçaram a hipótese estabelecida entre os índices de medo de errar e o tipo de vínculo profissional vivido pelos indivíduos e sinalizam a necessidade da adequação do construto postulado por Conroy (2002) ao ambiente organizacional brasileiro.

\section{Palavras chave}

Medo de errar; erro; medo organizacional. 


\section{Abstract}

Souza, Daniel Oswaldo Santana de; Tomei, Patricia Amélia (Advisor). The Fear of Failure at the Workplace. Rio de Janeiro, 2012. 70p. MSc. Dissertation - Departamento de Administração, Pontifícia Universidade Católica do Rio de Janeiro.

Many are the factors that contribute to the wide spreading of fear in the workplace. Technological changes and constant transformations on the management systems have been raising the pressure for excellence and productivity, intensifying the professional experience (CUNHA, 2006). Such scenario, in most of cases is acknowledged by researchers as something evil, capable of jeopardizing psychological and even physical integrity of those who experience it, negatively effecting organizational development (SUAREZ, 1993, APPLEBAUM, 1998). However, there are those who see in fear something positive. Such feeling, sometimes, when well managed is defined as a cornerstone for social interaction (KOURY, 2002) or even as a performance improver, helping managers and employees in a company. The main goal of this dissertation is to explore how demographic variables - such as age, gender, professional status, professional contract type and hierarchal level - effect on the intensity of the fear of failure in the workplace. In order to accomplish that, the Performance Failure Appraisal Inventory - PFAI (CONROY, 2002) was applied to an intentional, nonprobabilistic sample, chosen by accessibility of Business Administration graduate students from a university from Rio de Janeiro. Based on data statistical treatment, it could be observed "false low" scores, that highlight some limitations related to the expression of feelings, specially the negative ones, like fear. Besides the low scores measured for fear of failure, the descriptive statistics refused the hypothesis established between fear of failure and the professional contract type variable and indicate the need for fitting of the fear of failure construct (CONROY, 2002) to the Brazilian workplace.

\section{Keywords}

fear of failure; failure; organizational fear 


\section{Sumário}

1. Introdução 14

1.1. Objetivos 15

1.2. Relevância 15

1.3. Delimitação do Estudo 16

1.4. Estrutura do Estudo 16

2. Referencial Teórico 19

2.1. Medo 19

2.2. Comportamento Organizacional 22

2.3. Medo Organizacional $\quad 28$

2.4. Medo de Errar 33

2.5. Performance Failure Appraisal inventory (PFAI) 36

2.6. As Variáveis de Análise 38

2.6.1. Idade $\quad 38$

2.6.2. Gênero 39

2.6.3. Status Profissional 40

2.6.4. Vínculo Profissional 40

2.6.5. Hierarquia 41

3. Metodologia 42

3.1. Tipo de Pesquisa $\quad 42$

3.2. Universo e Amostra $\quad 42$

3.3. Coleta de Dados 43

3.4. Modelo Conceitual de Pesquisa 43

3.5. Hipóteses 45

3.5.1. Quanto à Idade 45

3.5.2. Quanto ao Gênero 46

3.5.3. Quanto ao Status Profissional 46

3.5.4. Quanto ao Tipo de Vínculo Profissional 46

3.5.5. Quanto ao Nível Hierárquico 46

3.6. Tratamento Estatístico $\quad 47$

3.7. Limitações do Método $\quad 47$

4. Resultados $\quad 48$

4.1. Perfil da Amostra 48

4.2. Confiabilidade do Questionário 50

4.3. Normalidade $\quad 50$

4.4. Teste de Hipóteses $\quad 50$

4.4.1. Quanto à Idade $\quad 51$

4.4.2. Quanto ao Gênero 51

4.4.3. Quanto ao Status Profissional 52

4.4.4. Quanto ao Tipo de Vínculo Profissional 53

4.4.5. Quanto ao Nível Hierárquico 54 
5. Conclusão 55

5.1. Do Cumprimento dos Objetivos 55

5.2. Limitações do Estudo

5.3. Sugestões para Trabalhos Futuros 58

6. Referência Bibliográfica $\quad 59$

Anexos $\quad 64$ 


\section{Lista de figuras}

Figura 1 - Indivíduo, grupo, departamento, organização 23

Figura 2 - Micro, macro 23

Figura 3 - Indivíduo(micro), grupo(meso), organização(macro) 24

Figura 4 - Medo em diferentes setores do sistema organizacional 30

Figura 5 - Erro, vergonha 33

Figura 6-Cinco dimensões de Conroy 35 


\section{Lista de tabelas}

Tabela 1 - Dimensão e questões relacionadas 44

Tabela 2 - Quanto ao pertencimento às coortes 49

Tabela 3 - Quanto ao posicionamento hierárquico dos
respondentes

Tabela 4 - Teste One-Sample Kolmogorov-Smirnov 50

Tabela 5 - O p-valor do teste Levene (quanto à idade) 51

Tabela 6 - O p-valor da ANOVA 51

Tabela 7 - O p-valor do teste Levene (quanto ao gênero) 52

Tabela 8 - O p-valor do teste Levene (quanto ao status

Tabela 9 - O p-valor do teste Levene (quanto ao tipo de vínculo profissional) 53

Tabela 10 - Descriptives 53

Tabela 11 - O p-valor do teste Levene (quanto ao nível
hierárquico)

Tabela 12 - O p-valor da ANOVA 54 


\section{Lista de gráficos}

Gráfico 1 - Distribuição da frequência das idades 
"O medo? O medo é um preconceito dos nervos. E um preconceito desfaz-se; basta a simples reflexão." Helena, Machado de Assis 